\title{
INVESTIGACIÓN
}

Recibido: 12/03/2021 --- Aceptado: 15/04/2021 --- Publicado: 24/05/2021

\section{ACTORES CLAVE Y FORMACIÓN EN EL SECTOR DEL TELEMARKETING. UN ESTUDIO DE CASO APLICANDO EL ANÁLISIS DE REDES SOCIALES}

\section{Key actors and training in the telemarketing sector. A case study applying the social networks analysis}

Julián Paniagua López ${ }^{1}$ Universidad de Valladolid. España.

julianantonio.paniagua@uva.es

\section{Cómo citar el artículo:}

Paniagua López, J. (2021). Actores clave y formación en el sector del telemarketing. Un estudio de caso aplicando el análisis de redes sociales.. Vivat Academia. Revista de Comunicación, 154, 321-341. doi: http://doi.org/10.15178/va.2021.154.e1354

\section{RESUMEN}

En este artículo se explica la organización del trabajo del sector del telemarketing. El objetivo es describir y analizar dos vulnerabilidades detectadas que afectan a la eficiencia y a la obtención del valor en las empresas de este sector. En primer lugar, se describirá la estructura comunicativa formal basada en las categorías profesionales del convenio colectivo, poniendo el centro de atención en la función de los actores clave de la organización y su línea de comunicación con los demás actores. La vulnerabilidad encontrada es la duplicación de funciones de los actores clave. En segundo lugar, se analizará el proceso formativo y las condiciones laborales de los teleoperadores. La vulnerabilidad es la deficiente formación de los trabajadores que afecta a la calidad del servicio. El método utilizado es el denominado análisis de redes sociales. Los datos reticulares se tomaron con la observación participante y se ha usado el programa informático UCINET para elaborar el correspondiente grafo. La conclusión es que ambas vulnerabilidades están íntimamente relacionadas porque pertenecen de manera integral a la organización del trabajo, afectan al buen funcionamiento diario y a la línea de comunicación tanto interna de los miembros de la empresa como a la externa de la empresa con los clientes.

\footnotetext{
${ }^{1}$ Julián Paniagua López. Es profesor de la Universidad de Valladolid. Especialista en análisis de redes sociales y sociología del trabajo. Ha realizado diversos estudios sobre cuestiones laborales. Destaca la monografía Las dos racionalidades. Estudio antropológico de una empresa textil.
} 
PALABRAS CLAVE: telemarketing - organización del trabajo - análisis de redes sociales - UCINET - actor clave - equivalencia estructural - taylorismo - formación línea de comunicación.

\begin{abstract}
This article explains the organization of work in the telemarketing industry. The objective is to describe and analyze two detected vulnerabilities that affect efficiency and the achievement of value in companies in this sector. First, the formal communication structure based on the professional categories of the collective agreement will be described, placing the focus on the role of the key actors in the organization and their line of communication with the other actors. The vulnerability is the duplication of functions of the key actors. Second, the training process and working conditions of telemarketers will be analyzed. The vulnerability is the deficient training of workers that affects the quality of the service. The method used is the so-called social network analysis. The reticular data were taken with the participant observation and the UCINET computer program was used to elaborate the corresponding graph. The conclusion is that both vulnerabilities are closely related because they belong in an integral way to the organization of work, they affect the good daily functioning and the line of communication both internally of the members of the company and externally of the company with clients.
\end{abstract}

KEY WORDS: telemarketing - work organization - social network analysis UCINET - Key actor - structural equvalence - taylorism - training - communication line.

\title{
1. INTRODUCCIÓN
}

El telemarketing es un servicio subcontratado que apareció tras la aplicación de las tecnologías de la información a la producción y la descentralización productiva. Se sustenta en la teoría de los recursos y capacidades, precursora de lo que se entendió después como gestión del conocimiento (Porter, 1982; Cuervo, 1995; Grant, 1996; Steward, 1997; Rimbau, 2002). Las empresas sólo realizan en su interior aquellos procesos que realmente aporten ventaja competitiva, subcontratándose el resto. Desde el conctact center se puede atender a los clientes con el consiguiente ahorro de tiempo y costes. Ha habido muchas críticas a este sector, comenzando por el consumidor, que muchas veces se ve impotente al no poder resolver su problema por teléfono; los trabajadores, por el estrés que produce este tipo de trabajo; hasta las propias empresas que contratan estos servicios, porque los empleados no siguen con rigor los procedimientos que se les exige. Sin embargo, debido al masivo uso de internet y los teléfonos móviles, junto con la posibilidad de realizar operaciones comerciales inmediatas independientemente de la distancia, las empresas necesitaron y necesitan el telemarketing porque resulta imposible disponer de una red de tiendas ubicadas en cada ciudad para atender a los clientes. Hacer eso supondría un enorme gasto y haría literalmente inviables a muchas empresas. 
A lo largo de estos años se han publicado varios trabajos en los cuales se describían algunos de los aspectos señalados. (Del Bono, 2000 y 2006; Belzunegui, 2002; Lara Rodríguez, 2003; Sáez, 2004; Montes, 2005; Calderón, 2006; Álvarez Ruiz, 2012; Ortega Olivares, 2014). Estos artículos se han centrado principalmente en las condiciones laborales de los trabajadores. No obstante, el interés por este sector ha disminuido en las revistas especializadas en las relaciones laborales, tales como Sociología del Trabajo o Cuaderno de Relaciones Laborales. Si en la página de Dialnet escribimos el término "telemarketing" nos aparecen siete páginas de referencias, sin embargo la mayoría de ellas corresponden a los años 90 del siglo pasado y la primera década de este, siendo escasos trabajos más recientes. Sí hay trabajos de sindicalistas (Pérez, 2014) y tesis doctorales (Carrasco, 2017). Desde la perspectiva empresarial destaca la revista Contact Center Hub, editada por Peldaño. Se edita desde el año 2000 con una frecuencia de 5 números al año. Los temas principales que desarrolla la revista son la tecnología, la expansión de las empresas, la relación con los clientes y el vínculo entre producción y emociones. En cualquier caso, en la literatura científica no abundan los trabajos con observación participante, aunque esta es una característica muy habitual en los estudios laborales, ni tampoco usando la metodología del análisis de redes sociales.

\section{OBJETIVOS}

El objetivo del artículo es explicar dos vulnerabilidades detectadas en la organización del trabajo que afectan a la eficiencia y a la obtención del valor en las empresas de este sector. En primer lugar, se describirá la estructura comunicativa formal basada en las categorías profesionales del convenio colectivo, poniendo el centro de atención en la función de los actores clave de la organización y su línea de comunicación con los demás actores. La vulnerabilidad encontrada es la duplicidad de funciones que puede conllevar algún conflicto de poder. En segundo lugar, se analizará el proceso formativo y las condiciones laborales de los teleoperadores. La vulnerabilidad encontrada es la deficiente formación de los teleoperadores y la permanente exigencia a la que se ven sometidos.

\section{METODOLOGÍA}

El método empleado para elaborar el artículo es el denominado análisis de redes sociales, basado en la teoría matemática de grafos (Wasserman y Faust, 2013). Como es sabido, en este método se usan diferentes programas informáticos especializados para recoger los datos, elaborar la matriz y construir finalmente el grafo que representa la forma de la red social estudiada. En este caso se ha empleado el programa UCINET (Borgatti, 2002). Los datos reticulares se han tomado de las propias empresas por medio de la observación participante, ya que el autor fue teleoperador durante tres años en diversas campañas, tanto de emisión como recepción, y en los últimos contratos se especializó en la banca telefónica. En el transcurso de la investigación se redactó un diario de campo escrito a mano, se hizo una media semanal de tres entrevistas semi estructuradas más las conversaciones en 
grupo. Soslayando las conversaciones, el número total de entrevistas fueron 176. Algunas de las empresas fueron Marktel Global Services S.A., Sitel Iberica Teleservices S.A., Unisono Soluciones de Negocio S.A., Global Sales Solutions Line S.L., Rainbow Comunicaciones S.L. todas ellas ubicadas en el municipio de Madrid.

Como es habitual en este método, lo primero es definir los nodos de la red y sus atributos para elaborar la matriz. Para ello se ha partido de las categorías profesionales que se señalan en el artículo 37 del Convenio Colectivo vigente (B.O.E. 12 de julio de 2017). Prácticamente se citarán literalmente para que se entienda mejor la función de cada nodo. Al final de cada una y entre paréntesis se indica el nombre de los nodos que aparecerán en el gráfico y con los que se ha elaborado la matriz. Son las siguientes:

Grupo profesional A.-Dirección o mandos superiores. Pertenecen a este grupo las personas que, por conocimiento o experiencia profesional, tienen atribuidas funciones directivas o de responsabilidad ejecutiva, coordinadora o asesora, con autonomía, capacidad de supervisión y responsabilidad, acordes con las funciones asignadas. Se incluyen en este grupo los directivos y responsables de departamento o área. (Director, presidente y jefe de operaciones).

Grupo profesional B.-Personal Técnico. Pertenecen a este grupo las personas que para el desarrollo de sus funciones deben tener una cualificación profesional en las técnicas propias del trabajo específico que desarrollan. Se incluyen en este grupo las personas con titulaciones superiores, titulaciones medias, y el personal en prácticas. (Comercial).

Grupo profesional C.-Personal Técnico en informática. Ejecutan de forma habitual las funciones propias de sistemas y desarrollos informáticos, reuniendo la cualificación adecuada para ellos. Se incluyen los jefes de proyectos, analistas, personal técnico de sistemas programación y ayudantes de sistemas. (Departamento de informática).

Grupo profesional D.-Administración y operación. Pertenecen a la administración las personas que, utilizando los medios operativos e informáticos, ejecutan de forma habitual las funciones propias administrativas de la empresa. (Administración y finanzas).

Pertenecen a la operación las personas encargadas de realizar tareas de operación de Contact Center, atendiendo o gestionando las llamadas y/o actividades administrativas, comerciales, relaciones públicas, organizativas, control de calidad, etc, bien individualmente o coordinando o formando a un grupo de ellas. Se incluyen en este grupo los jefes y jefas de administración, el personal técnico administrativo, oficiales, el personal auxiliar administrativo, responsables de servicio, el personal de supervisión, coordinación y formación, los agentes de calidad (quality), gestores, gestoras y personal teleoperador/operador en cualquiera de sus grados. (Formación, recursos humanos, supervisores, coordinadores y teleoperadores). 
Actores clave y formación en el sector del telemarketing. Un estudio de caso aplicando el análisis de redes sociales

Grupo profesional E.-Servicios generales. Carecen de cualificación y conocimientos especializados y sus funciones son auxiliares, tales como conserjes, ordenanzas, guardas y personal de limpieza. (No aparecen en la red porque son irrelevantes para los objetivos de este artículo y para la explicación del proceso productivo).

En definitiva, los actores de esta red son: presidente, director y jefe de operaciones que forman el grupo directivo. En términos de Mintzberg es el ápice estratégico (Mintzberg, 2000). Después están los departamentos técnicos: informática, recursos humanos, formación y administración y finanzas. En términos de Mintzberg corresponde a la tecnoestructura. Después los supervisores y coordinadores, que dirigen los equipos de trabajo, corresponde a la línea media. Y, por último, los teleoperadores, que forman núcleo de operaciones. Hay un último grupo de actores que se incluyen en la red denominados como "empresas" (A, B y C). Son las personas representantes de las empresas contratantes del servicio y que suelen estar físicamente en el edificio donde se realiza el trabajo. Según la propia organización pueden estar en un despacho independiente, pero también pueden estar en un cubículo en la misma sala donde están los teleoperadores.

El gráfico no representa una red real concreta de una empresa concreta, sino que es un modelo formal teórico, una síntesis de cómo está organizado este sector basado en la estructura organizativa del convenio colectivo. Salvo alguna particularidad propia que la dirección de la empresa aplique, en realidad todas las empresas de telemarketing funcionan básicamente igual, con las mismas categorías laborales, mismos salarios, misma organización formal y jerárquica y similares sistemas de incentivos, ascensos y disciplina. El diagrama es de tipo nominalista, en oposición al realista (Wasserman y Faust, 2013). En el gráfico saldrán siete nodos que son personas individuales: presidente, director, comercial, jefe de operaciones y los tres representantes de las empresas. Los coordinadores y los supervisores, por regla general son dos y uno respectivamente por campaña o equipo de trabajo, pero podría variar dependiendo del número de teleoperadores o la naturaleza del servicio. Se han puesto dos y uno para ajustarlo a la regla general. Los nodos de los departamentos de la tecnoestructura (informática, formación, recursos humanos y administración) son colectivos, en cada uno de ellos trabajan varias personas, pero no es necesario incluir a todas para explicar la organización formal del trabajo. Por último, los teleoperadores pueden ser desde uno solo hasta decenas de ellos en una sola plataforma. En el diagrama he incluido cinco por equipo de trabajo. Este número puede parecer arbitrario porque al igual que con los departamentos de la tecnoestructura, un solo nodo podría perfectamente representar al colectivo. Sin embargo, como demostraremos cuando se explique la parte cuantitativa, si pusiéramos un solo teleoperador no reflejaría la importancia estratégica que tienen los coordinadores. Los teleoperadores siempre son el grupo más numeroso y eso se tiene que reflejar en los datos estadísticos que el software proporciona. 
Paniagua López, J.

Actores clave y formación en el sector del telemarketing. Un estudio de caso aplicando el análisis de redes sociales

Los vínculos representan la doble dirección de la información, órdenes de trabajo y devolución del trabajo hecho, siguiéndose estrictamente ese orden jerárquico. El valor introducido en la matriz es un 1 para el caso de que exista vínculo entre los actores y un 0 para cuando no exista. Al representar solo la estructura formal no es necesario añadir más valores a los vínculos porque, como he indicado, cada par de actores recibe y envía información. En consecuencia, la red es simétrica y no valorada. A continuación, presento el diagrama general realizado con el programa UCINET:

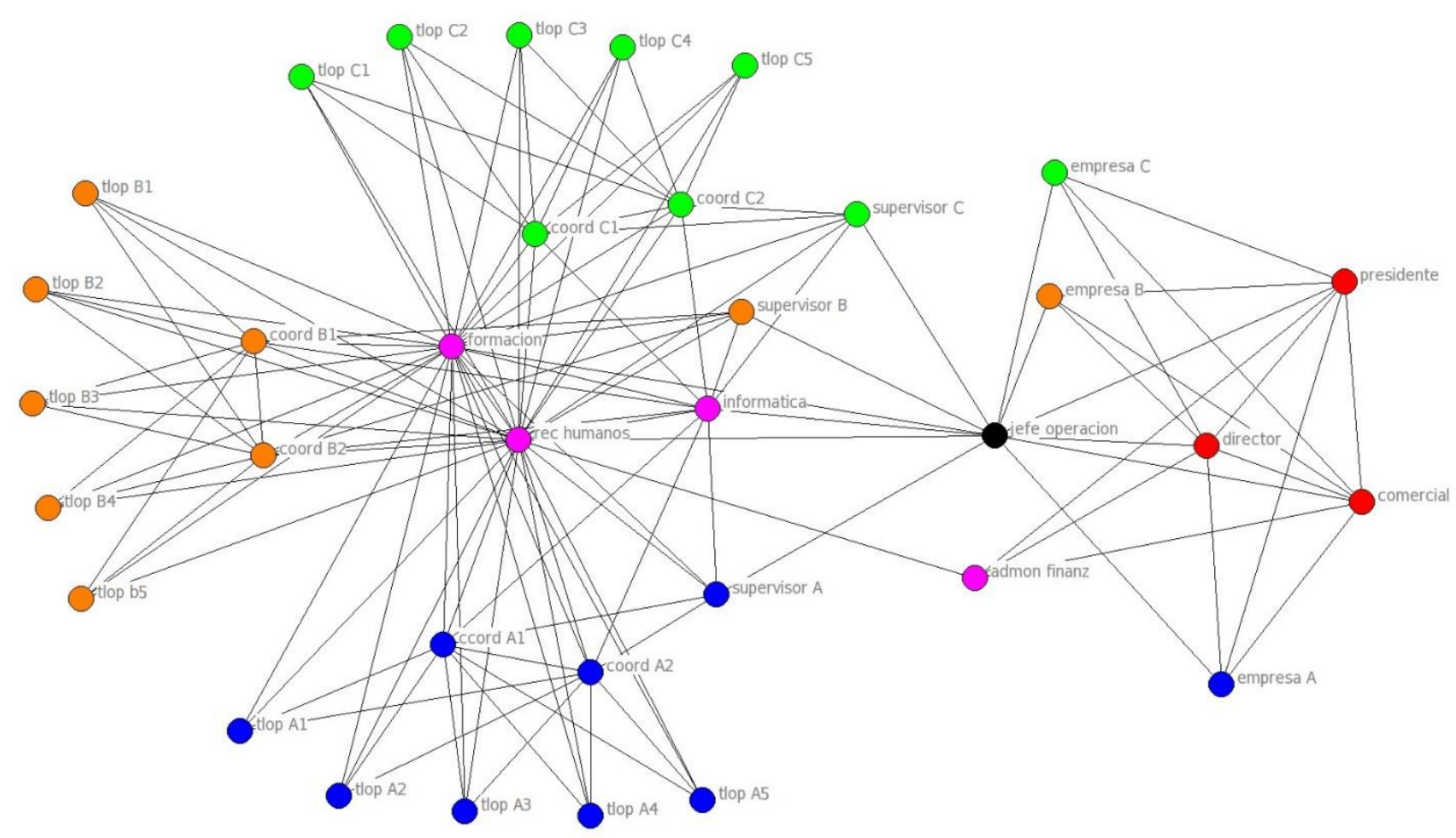

Figura 1. Grafo completo.

Fuente: Elaboración propia a partir de los datos de UCINET.

En el gráfico los grupos de trabajo están divididos por los colores, azul, marrón y verde corresponden a las campañas que puede haber en una plataforma. Los nodos rosas son los departamentos de la tecnoestructura (formación, recursos humanos, informática y administración) y los nodos rojos pertenecen al ápice estratégico. Queda el jefe de operaciones, que pertenece a la dirección pero que es un nexo que une al ápice estratégico con el núcleo de operaciones y con la tecnoestructura. No en todas las empresas denominan a esta persona con ese nombre. Puede ser denominado jefe de planta, director operativo de campaña, jefe de equipos, etcétera. Pero tanto la persona como su función existe en todas las empresas del sector.

Podemos observar la separación de los grupos de trabajo, que incluso físicamente están así ubicados en la plataforma. También observamos que hay dos nodos que pueden ser puntos de corte, el jefe de operaciones y la administración y finanzas. Aunque en este gráfico solo se representan las órdenes de trabajo formales, sin 
embargo, hay muchas más interacciones. Por ejemplo: si nos fijamos en el director vemos que solo se relaciona con siete nodos. La realidad es que puede relacionarse con más, como con los supervisores o con los otros departamentos, pero no es lo habitual porque delega en el jefe de operaciones. Lo mismo ocurre con las empresas, que pueden hablar con los supervisores, pero por regla general se respeta la jerarquía y esa comunicación pasa por el jefe de operaciones. La comunicación informal es relevante de cara a la ejecución del trabajo, especialmente si hay alguna incidencia durante la jornada, por la ayuda mutua entre los teleoperadores, pero es irrelevante desde el punto de vista de las órdenes de trabajo, ya que en el caso de que se deba modificar algún procedimiento se sigue la estructura jerárquica establecida.

\section{DISCUSIÓN}

La campaña se organiza de la siguiente forma: La empresa contratante del servicio impone las directrices de trabajo. Éstas dependen de la naturaleza del servicio, ya que no es lo mismo la revisión de las facturas que la venta de un seguro, por poner dos ejemplos. Se pacta el precio de cada llamada, su tiempo, el número de teleoperadores, los conocimientos requeridos, el horario, las formas de expresión, los objetivos a alcanzar, los incentivos salariales y las posibles sanciones. La empresa de telemarketing debe aportar el sistema informático, el software de gestión de las llamadas, el espacio con su mobiliario y contratar a los trabajadores. El software específico del servicio lo puede proporcionar la empresa contratante. Por ejemplo, si es un banco los teleoperadores usarán el mismo que el que se usa en las sucursales. La formación está remunerada y conlleva la firma de un documento de confidencialidad para no divulgar la información que manejen. En el caso de no firmarlo no se inicia el curso.

Tras la firma del contrato entre ambas empresas, el director y el representante de la empresa se lo comunican al jefe de operaciones. Éste le traslada todas las directrices y objetivos al supervisor de la campaña. También pueden reunirse la empresa, el director, el jefe de operaciones y el supervisor. Éste posteriormente informa a los coordinadores, que adquieren la formación correspondiente del producto. Después el jefe de operaciones y el supervisor hablan con los departamentos de informática, recursos humanos y formación para contratar y formar a los teleoperadores. La campaña comienza inmediatamente después del proceso de formación. Si es una campaña ya iniciada los nuevos teleoperadores se incorporan a la plataforma. La tecnología permite el control absoluto del trabajo.

La comunicación es siempre jerárquica de un estamento a otro, comenzando desde la dirección hacia los teleoperadores (órdenes de trabajo) y devolviéndola (resultados del trabajo). Esta segunda categoría de información se divide en dos tipos, los resultados de la campaña y el rendimiento de los trabajadores. Continuamente a lo largo de la jornada los coordinadores van recibiendo los resultados del trabajo: número de llamadas atendidas, minutos empleados, ventas (si la campaña es de ventas) y resolución de las consultas e incidencias. Esa información se la entrega al supervisor, después al jefe de operaciones que a su vez se la entrega al representante 
de la empresa y al director. El segundo tipo de información corresponde al rendimiento de los trabajadores y contiene varios aspectos. Uno de los principales es la relación entre el número de llamadas atendidas y el tiempo empleado. Hay campañas cuyas llamadas no deben superar los 4,5 minutos de duración. Esto sería el rendimiento óptimo. Si en la media total durante el periodo de un mes el teleoperador ha superado ese tiempo, entonces puede ser despedido. Otros aspectos que se evalúan son las ventas alcanzadas, la calidad y satisfacción de la atención, la actitud desempeñada, el tipo de palabras o expresiones empleadas, la capacidad de resolución de los conflictos, el tiempo que se tarda en la búsqueda de información, el tiempo de espera de las llamadas y el desempeño general en el trabajo. Esta información la obtienen los coordinadores y se la envían al supervisor, que posteriormente se la entrega primero al jefe de operaciones $\mathrm{y}$, después, a recursos humanos y estos a su vez al departamento de administración y finanzas.

Todo queda registrado: la conversación completa, que se graba y funciona como contrato con valor jurídico en el caso de una venta; lo que se escribe en el ordenador; y los minutos en que los teleoperadores están desconectados y su causa. Esta información se guarda en los servidores del sistema informático y la gestionan los coordinadores, que pueden monotorizar en cualquier momento tanto las pantallas como las conversaciones de los teleoperadores. Todo está medido, calculado y vigilado. No hay autonomía en el trabajo, excepto la de algunas palabras usadas, pero incluso eso está regulado porque, por regla general, no se permite el uso de expresiones coloquiales que faciliten el entendimiento con los clientes, sino que se enseña a usar los eufemismos. Es la aplicación absoluta del taylorismo: el control total de las tareas (palabras, tono, escritura y tiempo); la vinculación de una tarea con un tiempo de ejecución; y, finalmente, la separación radical de concepción y ejecución (Braverman, 1978; Braga, 2006; Pena, Cardim y Araújo, 2011).

A continuación, haremos un breve análisis de los datos estadísticos que nos aporta UCINET a través de los conceptos de grado, cercanía e intermediación. El análisis del grado (número de vínculos directos que tiene cada nodo) no ofrece mucha dificultad. Solo hay que ver el diagrama para darse cuenta que los nodos formación y recursos humanos son los que más vínculos poseen, seguidos del jefe de operaciones, informática y los coordinadores. Sin embargo, estos datos no son tan relevantes de cara a la explicación de la organización del trabajo, porque desde el punto de vista operativo, ya iniciada la campaña la participación de los departamentos de formación y recursos humanos se reduce. Puede haber cursos formativos o nuevas contrataciones, pero no intervienen en las tareas, excepto para recibir los resultados del trabajo. El departamento de informática es más relevante, ya que sin él sería imposible la actividad, pero su trabajo consiste en que el sistema funcione correctamente. En realidad, el principal valor de la empresa, lo que puede o no generar beneficios, es la relación de los teleoperadores con los clientes. Desarrollaré esta idea más adelante. Si eliminamos esos dos nodos del gráfico observamos el flujo de comunicación más simplificado: 
Paniagua López, J.

Actores clave y formación en el sector del telemarketing. Un estudio de caso aplicando el análisis de redes sociales

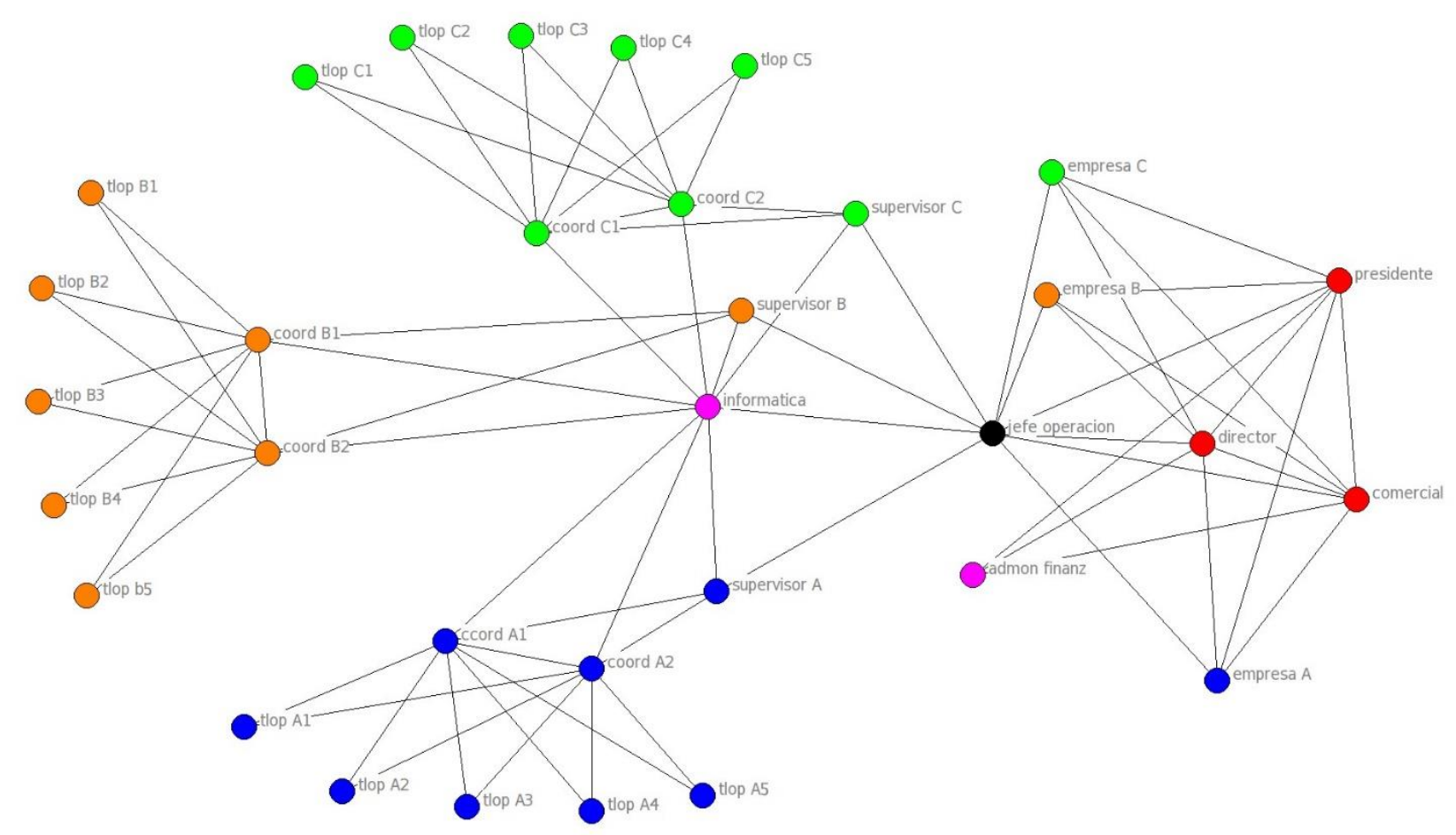

Figura 2. Grafo sin los nodos Recursos Humanos y Formación.

Fuente: Elaboración propia a partir de los datos de UCINET.

Todo pasa por el jefe de operaciones, que es un punto de corte de la red. Es, además, el nodo con mayor número de vínculos directos, 11, seguido por el informático, 10, los coordinadores, 8, el director, 7, empresas y supervisores, 4. Este gráfico representa la estructura organizativa una vez que ya se ha iniciado la campaña y los teleoperadores están recibiendo llamadas.

La cercanía mide las distancias geodésicas de todos los nodos. Cuanto menor sea la distancia de un nodo con los demás, más cercano estará con respecto a ellos, por lo tanto, tendrá mayor índice de centralidad. Y a la inversa, cuanto mayor sea la distancia geodésica, más periférico será. Los datos de los nodos más centrales son los siguientes:

Tabla 1. Medida de la cercanía (versión reducida).

\begin{tabular}{llll}
\hline & & 1 & 2 \\
\hline & & Farness & nCloseness \\
8 & Rec. humanos & 40.000 & 85.000 \\
9 & formación & 41.000 & 82.927 \\
11 & Jefe operación & 56.000 & 60.714 \\
10 & informática & 56.000 & 60.714 \\
20 & Supervisores (todos) & 62.000 & 54.839 \\
\hline 22 & Coordinadores (todos) & 64.000 & 53.125 \\
\hline
\end{tabular}


Paniagua López, J.

Actores clave y formación en el sector del telemarketing. Un estudio de caso aplicando el análisis de redes sociales

Fuente: Elaboración propia a partir de los datos de UCINET.

Independientemente de que en las estadísticas vuelvan a aparecer recursos humanos y formación como los nodos más cercanos a los demás, en este caso lo relevante es que son el jefe de operaciones y los supervisores los nodos centrales, seguidos de los coordinadores, justo los actores que realmente dirigen el trabajo. Son los que más información de primera mano manejan durante la jornada. Aunque el ápice estratégico está en la periferia de la red y la información que reciben es indirecta y delegada, ello no implica que su capacidad de mando se reduzca. En el caso de que un subordinado manipule o tergiverse los datos se arriesga a perder su puesto de trabajo o incluso a una demanda judicial. Y si no se alcanzan los resultados esperados, las empresas o el director pedirán las correspondientes explicaciones.

La intermediación mide las veces que un nodo se interpone entre otros en su distancia geodésica. Los datos son los siguientes:

Tabla 2. Medida de la intermediación (versión reducida).

\begin{tabular}{llll}
\hline & & 1 & 2 \\
\hline 8 & Rec. humanos & Betweenness & nBetweenness \\
9 & formación & 205.700 & 36.667 \\
11 & Jefe operación & 160.850 & 28.672 \\
7 & Admin finanz & 143.400 & 25.561 \\
10 & informática & 20.100 & 3.583 \\
\hline 14 & Coordinadores (todos) & 18.350 & 3.271 \\
\hline 20 & Supervisores (A, B y C) & 5.000 & 0.891 \\
\hline 1 & presidente & 3.200 & 0.570 \\
\hline 2 & director & 2.000 & 0.357 \\
\hline 3 & Comercial & 2.000 & 0.357 \\
\hline 15 & Teleoperadores (todos) & 2.000 & 0.357 \\
\hline 19 & Empresas (A, B y C) & 0.000 & 0.000 \\
\hline
\end{tabular}

Fuente: Elaboración propia a partir de los datos de UCINET.

De nuevo los nodos pertenecientes a la tecnoestructura y el jefe de operaciones son los mediadores a través de los cuales circula la mayor parte de la información. Sin embargo, aquí hay que destacar la relevancia de los coordinadores. En la literatura sobre gestión empresarial está muy asentada la importancia de los mandos intermedios de cara a la eficiencia de la organización (Alfaro Escolar, 1991). Son quienes más presión reciben por parte del supervisor si no se alcanzan los resultados. Pero también son los que gestionan la información de primera mano, tanto cuantitativa como cualitativa. Conocen el estado de ánimo de los teleoperadores, sus capacidades reales, saben quién y como puede resolver las incidencias, a quien elegir para una necesidad puntual, quien puede ayudar y enseñar mejor a los novatos 
durante su periodo de formación e incluso pueden defender a los teleoperadores de algún error que pudieran cometer, siempre y cuando sea defendible. Dos ejemplos. Un error defendible es el uso de alguna palabra o expresión inadecuada en una conversación con un cliente, como puede ser un término vulgar cotidiano. Esas palabras quiebran la denominada excelencia telefónica y no da buena imagen. El teleoperador es amonestado y si ya ha demostrado eficiencia el error no pasa de ahí, pero no se puede repetir. A un novato no se le consiente ese tipo de lenguaje. Un error no defendible es si al cliente se le da una información errónea. Si reclama se coteja la grabación de la conversación y si tiene razón la empresa está obligada a indemnizarle. La indemnización la paga la empresa de telemarketing. El teleoperador recibe una amonestación y según sea la cantidad puede pagarla con los incentivos a los que pudiera tener derecho o incluso se le puede despedir si la cantidad es muy alta. Un error de este tipo moviliza a toda la plataforma, especialmente a los coordinadores y al departamento de formación, porque lo que está en juego no es solo una indemnización puntual, sino el servicio mismo, ya que la empresa puede perder la confianza por la mala formación de los teleoperadores. Por este tipo de casos, junto con los datos del rendimiento, los coordinadores son los verdaderos mediadores.

A la luz de los datos cuantitativos que nos proporcionan las medidas de centralidad, una pregunta pertinente sería si los departamentos de la tecnoestructura ejercen algún tipo de influencia en la organización y cómo sería esa influencia. La respuesta es no o muy escasa. Y esto es así por las funciones propias de cada departamento. Recursos humanos se encarga de seleccionar a los trabajadores, elaborar los informes de rendimiento y otorgar los incentivos salariales si existen y se han alcanzado los resultados para merecerlos. Pero toda esta información se la proporcionan los coordinadores. El departamento de formación cumple su función formativa y motivadora. El de informática está para que el sistema funcione correctamente y, por último, el departamento de contabilidad se dedica a las cuentas de la empresa. Ninguno de estos cuatro departamentos toma decisiones estratégicas. Podrían ejercer alguna influencia desde el ámbito informal debido a la cantidad de información que manejan, pero incluso así seguiría siendo muy limitada, ya que no son mano de obra directa. Las normas de este sector son muy rígidas $\mathrm{y}$, lo más importante, es muy difícil tergiversar los datos porque todo queda registrado y hay varios caminos por donde circula la información. No obstante, hace unos años sí era posible esa manipulación de los datos del rendimiento.

Los coordinadores son quienes pueden ejercer alguna influencia desde la organización informal. ¿Cuál? Otorgar algún tipo de favoritismo hacia unos teleoperadores en detrimento de otros, en dos aspectos: en primer lugar, de cara tener un buen rendimiento para cobrar los incentivos salariales; en segundo lugar, de cara a una posible promoción en las convocatorias a coordinador. Ahora bien, ese favoritismo hay que justificarlo documentalmente. ¿Cómo? Manipulando el documento de excell que diariamente se genera durante el transcurso de la jornada. Aquí hay que señalar una evolución en la tecnología. Esto se podía hacer durante los primeros años del auge del telemarketing, entre 2000 y 2008. El coordinador que 
quería favorecer (o también despedir) a algún teleoperador podía reescribir ese documento y tanto al supervisor como a recursos humanos llegaba con los datos inventados. Hacia 2008 comenzaron a aparecer otros programas informáticos que registraban los datos según se iban produciendo, a los cuales tenían y tienen acceso el supervisor, el jefe de operaciones e incluso el delegado de la empresa cliente, con lo cual esa manipulación es ya muy difícil de hacer, por no decir imposible. Las principales empresas de telemarketing poseen software de este tipo.

Pero a pesar de la tecnología y de los rígidos procedimientos administrativos se puede favorecer arbitrariamente a algún teleoperador en detrimento de los demás: ubicando a la persona favorecida en una campaña en la cual es más fácil alcanzar los resultados exigidos. Pongo un ejemplo de mi propia experiencia. Durante ocho meses trabajé para un banco on-line. El equipo lo formábamos dos coordinadores y 24 teleoperadores distribuidos en dos turnos de trabajo y en tres campañas de emisión. Dos de ellas eran difíciles porque había que ofrecer diversos productos del banco y comprobar los datos de una tarjeta de consumo para un gran centro comercial. El trabajo en ambas campañas era monótono y repetitivo y las conversaciones con los clientes no siempre eran amables. La otra campaña estaba dirigida a los clientes del banco con mucho dinero y previamente seleccionados, a los cuales se les ofrecía productos con altas posibilidades de beneficio, planes de ahorro, fondos de inversión, etcétera. Las conversaciones con ellos eran con tono amable. Debido tanto al tipo de producto como a las características sociológicas de los clientes, era relativamente fácil alcanzar un buen rendimiento en el trabajo sin necesidad de manipular ningún documento. Dentro del equipo solo había rotación en las dos primeras campañas mientras que en la tercera siempre la hacían dos chicas, que eran amigas del coordinador. Todos los meses alcanzaban los resultados exigidos, por lo que cobraban los incentivos y a una de ellas la promocionaron para coordinadora. Los demás teníamos que continuar haciendo el trabajo monótono y repetitivo. Cuando alguna vez planteamos hacer rotación en todas las campañas, el coordinador justificaba su negativa diciendo que las dos chicas habían demostrado suficiente eficiencia y cuando los demás alcanzáramos el mismo nivel se podría comenzar a rotar (sic).

El último de los criterios para analizar esta red es la equivalencia estructural. Dos actores son equivalentes si tienen idénticos vínculos con los demás, por tanto, son sustituibles. Con ello entramos en la primera de las vulnerabilidades mencionadas al principio del artículo, la del actor clave de la red. Siguiendo la definición de Borgatti (Borgatti, 2013), hay dos criterios para determinar a un actor clave. El primero es que si se elimina ese nodo se perturbaría al máximo la comunicación con los nodos restantes. Es decir, es un punto de corte que puede desconectar la red. El segundo es que ese nodo está conectado al máximo con todos los demás. Hemos observado que en esta red hay que eliminar a dos nodos para desconectarla, el jefe de operaciones y el departamento de finanzas. Pero desde el punto de vista operativo el relevante el jefe de operaciones. Asimismo, el nodo con más vínculos es recursos humanos. Pero ya se ha indicado que a efectos operativos la relevancia de este actor se reduce. Por lo tanto, el actor clave es el jefe de operaciones. Es un puesto de total confianza porque 
Actores clave y formación en el sector del telemarketing. Un estudio de caso aplicando el análisis de redes sociales

con la información que recibe debe motivar o presionar a los supervisores y a los coordinadores para alcanzar los resultados y explicar a las empresas la marcha de la campaña, las dificultades e incidencias que ocurren y dar pautas para modificar algún procedimiento, todo ello basado en los datos reales que debe saber analizar e interpretar. Es, en definitiva, un líder.

Para el análisis de la equivalencia estructural hemos usado la medida denominada convergencia de correlaciones iteradas (CONCOR), que mide la similitud de las relaciones entre los actores y los divide en subconjuntos o posiciones, de tal manera que los actores dentro de una posición son más cercanamente equivalentes entre ellos. Aparte de la tabla de datos estadística, esta medida proporciona un diagrama de árbol que va uniendo los nodos. Los que están conectados en la parte izquierda están muy cerca de tener una equivalencia estructural perfecta, mientras que los subgrupos de actores que están unidos sólo a través de caminos en la parte derecha del diagrama tienen menor equivalencia estructural o ninguna. El diagrama que nos proporciona UCINET es el siguiente:

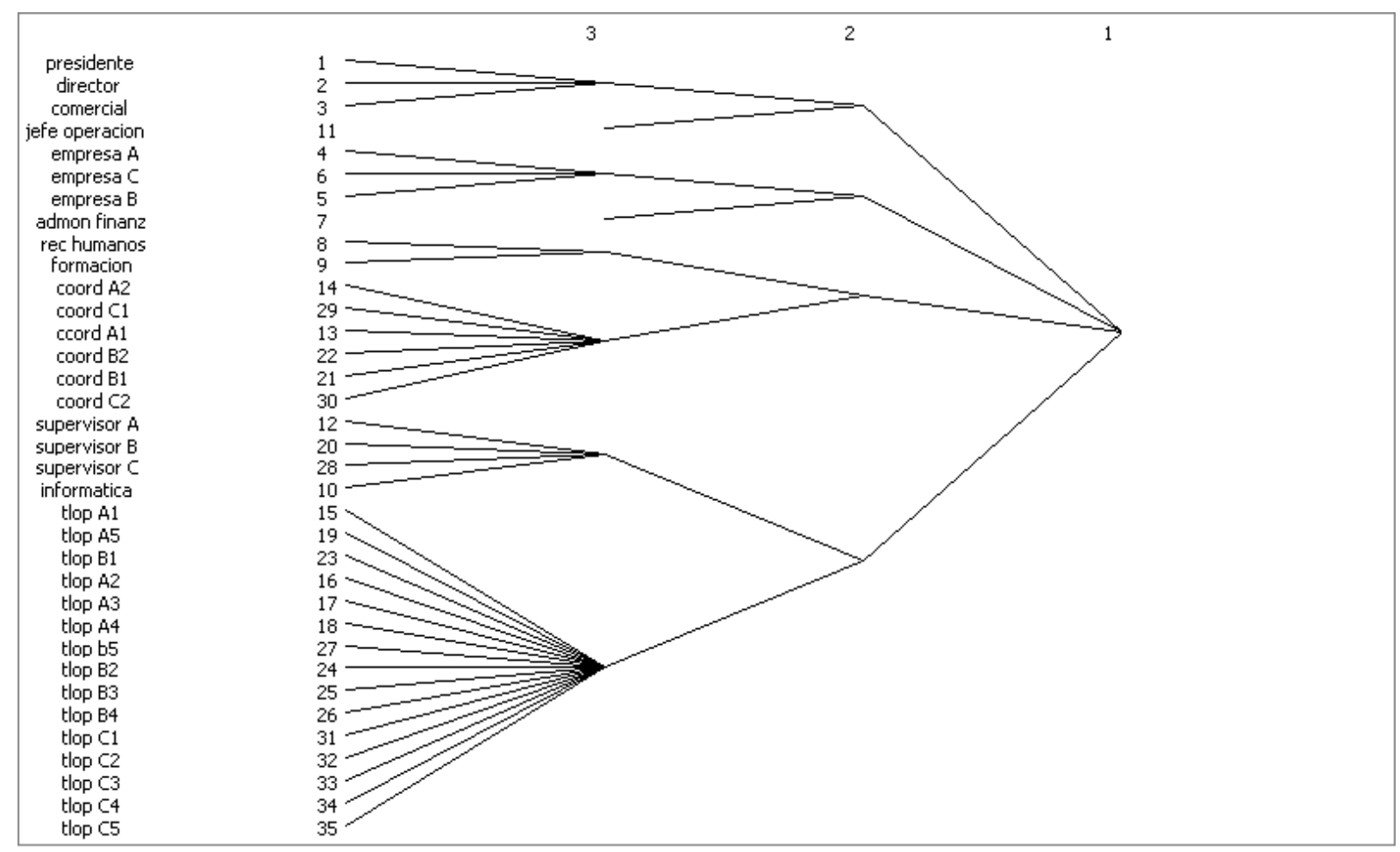

Figura 3. Equivalencia estructural CONCOR.

Fuente: Elaboración propia a partir de los datos de UCINET.

En total son ocho posiciones. Siguiendo la definición de actor clave, debemos incluir a los supervisores. Y lo son no solo por sus funciones operativas y de enlace entre los grupos de trabajo y la dirección, sino porque, aunque no son exactamente puntos de corte, si se eliminan perturbarían parte de la comunicación entre los nodos restantes. Observemos el siguiente gráfico: 
Paniagua López, J.

Actores clave y formación en el sector del telemarketing. Un estudio de caso aplicando el análisis de redes sociales

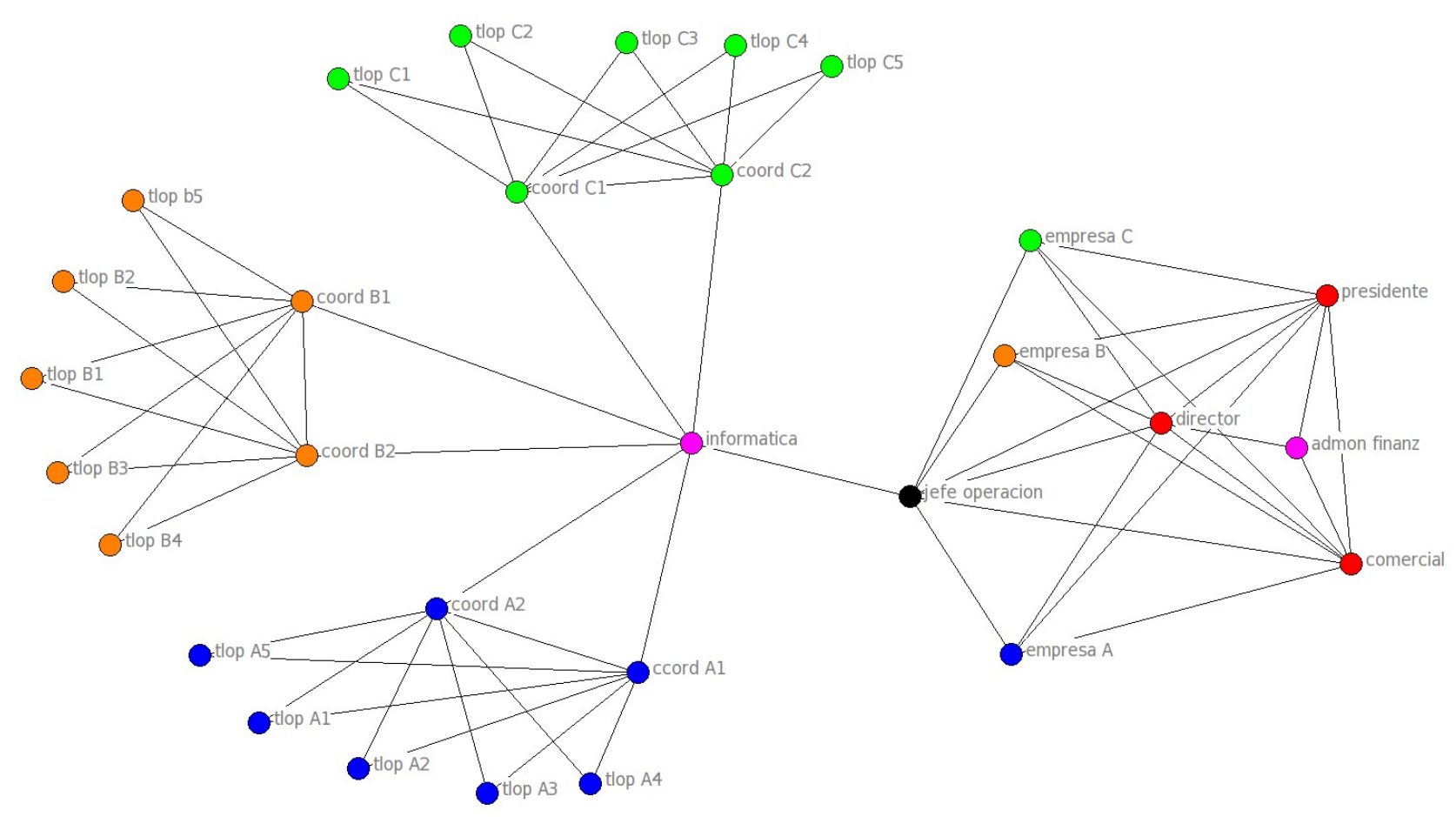

Figura 4. Grafo sin los nodos Recursos Humanos, Formación y supervisores.

Fuente: Elaboración propia a partir de los datos de UCINET.

Al eliminar los nodos de formación, recursos humanos y a los supervisores podemos comprobar la debilidad a la que aludíamos al principio: los caminos de la información, aunque pasan por el departamento de recursos humanos, sin embargo, no son del todo suficientes para que lleguen al ápice estratégico. Llega la información cuantitativa elaborada minuto a minuto a partir de las llamadas atendidas, pero no toda la información cualitativa, la del contenido de las conversaciones telefónicas. Por lo tanto, habría que establecer otras líneas de comunicación entre los teleoperadores y la dirección.

La primera solución sería incrementar los vínculos del director, de tal manera que este y el jefe de operaciones fueran equivalentes. La segunda solución sería que entre el representante de la empresa y el supervisor se creara un vínculo. En la práctica sí se comunican, pero como se ha indicado es muy puntual y siempre está mediado por el jefe de operaciones. No obstante, ambas soluciones generarían a su vez un problema que ya no sería técnico, sino político, porque al analizar la equivalencia estructural se descubre que entre los tres nodos (supervisor, jefe de operaciones y director) al menos uno sobra, ya que sus funciones las puede realizar otro o están duplicadas. Si hubiera un vínculo formal entre la empresa y el supervisor, entonces sobra el jefe de operaciones. A su vez, si el director tuviera los mismos vínculos que el jefe de operaciones, se duplicarían sus funciones. Para empeorar más la situación, las tareas de análisis de los resultados del trabajo y elaborar los informes para la empresa las pueden hacer los supervisores e incluso los coordinadores, que son quienes reciben de primera mano toda la información, tanto cuantitativa como 
cualitativa. La aplicación de la equivalencia estructural descubre que hay al menos dos personas con las mismas funciones, pero con diferente salario y diferente poder, el supervisor y el jefe de operaciones por un lado y éste y el director por otro. En este sector suele haber este tipo de problemas de comunicación e incluso rivalidades y a veces es la causa del fracaso de una campaña o pérdida del contrato de servicios.

Aunque estos conflictos no suelen salir de los despachos y son difíciles de demostrar, sin embargo, se conocen pasado un tiempo por algunas conversaciones de los supervisores, de los trabajadores de recursos humanos y de finanzas que lo cuentan a personas de su máxima confianza. En el trabajo con el tiempo todo acaba sabiéndose porque la comunicación informal siempre se produce, aunque no de manera inmediata. No obstante, estos problemas de poder y celos profesionales se detectan por las órdenes contradictorias que en un momento dado reciben los supervisores y los coordinadores y se las trasladan a los teleoperadores. Por ejemplo, un cambio repentino en algún procedimiento y un tiempo después, que según el caso pueden ser unos minutos o unas horas, una contraorden que revoca la primera. El origen de ambas procede de la diferente interpretación de las directrices de la empresa que han tenido el director y el jefe de operaciones. Estas disfunciones ocurren con más frecuencia en las campañas de telefonía móvil que en las de los bancos.

Por último, vamos a explicar la segunda vulnerabilidad mencionada, el sistema de formación de los teleoperadores. Cualquier trabajo, por simple y desespecializado que sea, exige unos mínimos conocimientos. El trabajo de un teleoperador es una combinación de tres aprendizajes: las características del producto, los programas informáticos y el uso del lenguaje adecuado, por ese orden. Aunque la mayoría de los clientes desconozcan las características de los productos, sin embargo los teleoperadores deben conocerlas porque en cualquier momento alguien puede preguntar por ellas. Por ejemplo: en una tarjeta bancaria están los datos del cliente, las cláusulas del contrato, los servicios que presta, la interacción técnica con los cajeros o con los puntos de pago, el procedimiento técnico y administrativo para realizar las operaciones, las leyes, la ubicación geográfica de donde se haya usado y las operaciones realizadas, por solo citar algunos datos que aparecen en la pantalla del teleoperdor. Toda esa información aparece en una sola pregunta y hay que buscarla por los diferentes menús, pantallas o incluso abrir varios programas para responder con precisión y rapidez. Esta complejidad cognoscitiva se divide en cuatro aspectos que el teleoperador debe desarrollar de manera integral durante el transcurso de la llamada: a) debe conocer las características del producto; b) debe manejar con soltura los programas informáticos; c) debe emplear el lenguaje adecuado, usando los términos específicos del producto, pero dando las explicaciones pertinentes para que el cliente entienda lo que se le dice, adaptándose a su nivel intelectual, sin perder el rigor profesional y manteniendo la corrección y educación en la conversación; y d) no debe sobrepasar el tiempo estipulado en la llamada, porque cuanto más tiempo tarde en resolverla, menos productivo es $\mathrm{y}$, a la vez, menos llamadas se atienden porque quedan en espera. La parte más difícil es la c, porque si el cliente no entiende la respuesta a su consulta, o no está conforme con 
la explicación, o su manera de expresarse es muy confusa y desordenada, el teleoperador tiene que incrementar sus esfuerzos, capacidades intelectuales y retóricas para que el cliente entienda el procedimiento y sus causas. Por supuesto no debe emplear un lenguaje ofensivo, debe expresar amabilidad incluso con clientes que le insultan. Hay que añadir que el tiempo de silencio entre el final de una llamada y el comienzo de la siguiente no es mayor de cinco segundos. Para alcanzar un rendimiento aceptable se necesita más de un mes y mucha ayuda de los coordinadores y los compañeros veteranos.

Estas dificultades se podrían resolver con una buena enseñanza y ampliando el tiempo de práctica. Los departamentos de formación suelen decir que forman bien a los nuevos teleoperadores y que es continua. Hay que disentir plenamente de esta afirmación, pero no porque los profesores desconozcan las características del producto que explican, sino por tres errores que se cometen durante el curso: la denominada "escucha activa", la confidencialidad y la equiparación entre el tiempo de formación con el tiempo de producción.

La formación en el telemarketing parte de un principio pedagógico erróneo: se considera que si dan al alumno un mensaje y éste tiene lo que denominan "escucha activa", ya tiene que asimilarlo y retenerlo en la memoria. Dicho una vez, ya tiene que quedar aprendido. Eso sería posible si los datos fueran pocos y la relación entre ellos y los procedimientos a seguir fueran sencillos. Pero en servicios complejos como la banca, los seguros o la facturación, la cantidad de información que reciben los alumnos es tan enorme y las aplicaciones informáticas tan complejas, que al final del tiempo de aprendizaje de una sola jornada es imposible recordar lo explicado en la primera hora, y lo que se ha dicho el lunes, el viernes se ha olvidado. Algunas campañas tienen un periodo de formación de un mes completo impartiendo clases entre seis y ocho horas diarias. Obviamente hay repasos, pero al ser todo teórico expuesto con diapositivas es muy difícil estructurar los apuntes tomados y organizar esa información en el cerebro. Cuando los alumnos piden que se repita algo los profesores lo repiten, pero también es muy frecuente que añadan la coletilla "esto ya lo expliqué" y recuerdan continuamente lo de la "escucha activa". Las clases prácticas con el ordenador y con todos los programas que se usan también son muy escasas. Debido a esto, cuando el teleoperador está ya conectado al sistema y recibiendo llamadas, al principio ni siquiera sabe dónde pinchar. Es cierto que en clase se han visto las pantallas y los diferentes menús que tienen los programas, pero no sirve de nada que el profesor explique las opciones que tiene un menú o qué casilla hay que pinchar para activar un servicio si eso solo se ve en la diapositiva, algunas de ellas solo una vez y no se hace en el ordenador. Es imposible memorizar el funcionamiento de un programa informático si no se practica con frecuencia, especialmente con algunos programas, como los de facturación, que tienen la pantalla dividida en tres grupos de menús y para llegar a activar una opción hay que seguir una ruta de al menos cuatro pasos. Eso no se aprende solo con diapositivas, hay que practicarlo continuamente. 
Tampoco se proporciona material de estudio, todo se basa en apuntes tomados en clase. A veces los profesores dan fotocopias de algún procedimiento o algún esquema, pero son muy escasos. Las empresas no dan el material por motivos de confidencialidad de la información, a pesar de firmar ese documento. Aparte de cumplir la ley de protección de datos, lo que ocurre (esto dicho por lo propios profesores) es que las empresas no quieren que se divulgue la información de sus productos, sus procedimientos y el software que utilizan. Si proporcionaran ese material a los alumnos y, después, por cualquier causa, ese trabajador no continúa, ese material ya estaría fuera de su control, por lo tanto evitan esa posibilidad en la medida de lo posible. La consecuencia es que es muy difícil retener los conocimientos. Ese material está en la intranet de la empresa y contiene las características de los productos, se detallan los procedimientos a seguir, la casuística, la información legal, qué hacer en caso de conflicto y preguntas frecuentes. Si los profesores explicaran a partir de estos documentos y los teleoperadores pudieran estudiarlos, la formación mejoraría en un altísimo porcentaje, se reduciría el estrés inicial y se perdería menos tiempo en consultar durante el transcurso de la llamada. Pero solo se puede acceder a la intranet cuando ya el teleoperador está en la plataforma y con el cliente al teléfono, con lo cual no le da tiempo atender la llamada y buscar información a la vez, tiene que dejar al cliente en espera. De hecho los novatos no saben ni cómo buscar la información y tienen que recurrir constantemente a los coordinadores que, a su vez, les recuerdan ya con más énfasis lo de la "escucha activa".

Hay que señalar también algunas importantes deficiencias pedagógicas de los profesores. Todos han sido previamente teleoperadores y conocen las características de los productos, pero no saben transmitirlo. Son muy pocos los que explican con orden, claridad y precisión los contenidos que se deben conocer y cómo aplicarlos luego en el puesto de trabajo. Algunos ni siquiera llevan un índice, dan algunas cosas por sabidas, no dan definiciones precisas e incluso saltan de un tema a otro sin continuidad y sin relación. El resultado es que los apuntes son caóticos y fragmentados. Esto lo comentan los teleoperadores con frecuencia, entre broma y preocupación, durante el tiempo de descanso. Para solucionar estas deficiencias algunos teleoperadores se reúnen o se comunican por internet y completan los apuntes unos con otros. Es una estrategia de auto ayuda porque en los cursos suele haber al menos dos exámenes. Si no se aprueba, no se continúa el proceso formativo.

Debido a que la formación está remunerada, el tiempo de formación es el mismo que el que se dedicaría a trabajar ya dentro de la plataforma. Esto significa que los descansos reglamentarios son de cinco minutos cada hora, que se unifican todos, con un descanso más largo de 20 minutos. En un curso de siete horas diarias solo se descansan 40 minutos, divididos en dos tiempos de 20, el resto del tiempo se está impartiendo clase dando información continua y desarrollando contenidos. Puedo asegurar que las dos últimas horas son horas perdidas porque la capacidad de atención se ha agotado. Sin embargo, los profesores siguen impartiendo los temas como si del primer minuto se tratase. 
No es lo mismo el tiempo de formación que el tiempo de producción. El proceso de aprendizaje y adquisición de los contenidos con la debida competencia requiere tiempo, repetición y ensayo. No hay prácticas reales después de explicar los temas, solo hay unos días de escuchas con un compañero veterano, por lo tanto la asimilación de los contenidos es muy débil. Y no estamos hablando de procesos que se puedan explicar con conceptos generales, a modo de explicación filosófica o sociológica de algún acontecimiento. Estamos hablando de datos concretos y precisos, con valor jurídico que se usan desde la primera llamada, como por ejemplo qué comisión tiene una tarjeta al usarse en el extranjero o a qué se debe el incremento del precio de la luz si el consumo ha sido el mismo que el mes anterior. La consecuencia es que los teleoperadores comienzan su trabajo con escasas competencias, pero con la exigencia de eficiencia completa desde el primer minuto. Esto genera altos niveles de estrés porque el cliente no sabe ni tiene por qué saber su situación, lo que quiere es que su demanda sea atendida con precisión y profesionalidad. Al final las competencias se adquieren con el tiempo, por ensayo y error y por la ayuda mutua entre todos los compañeros. Doeringer y Piore ya señalaron estas deficiencias formativas en su estudio sobre los mercados internos de trabajo (Doeringer y Piore, 1985). En última instancia la formación suele tener lugar en el proceso productivo, a través del ensayo y el error. También indicaban la diferencia entre explicar cómo hacer el trabajo y hacerlo efectivamente. Muchos trabajadores o mandos intermedios saben perfectamente como ejecutarlo, pero son incapaces de decirlo sin hacerlo. Esto implica un triple esfuerzo: los trabajadores tienen que esforzarse más durante su periodo de formación, los coordinadores tienen que dedicar más atención a los novatos sin dejar de cumplir su propio trabajo y todo ello supone un gasto para la empresa, porque durante la formación puede haber deterioro de material o errores que pueden provocar la pérdida de clientes. El pensamiento general de los teleoperadores es que la formación no existe, que se aprende equivocándose continuamente, recibiendo insultos de los clientes y alguna amonestación de los coordinadores, preguntando a los compañeros y espabilando a marchas forzadas, porque si en dos semanas no se ha avanzado lo suficiente, se tiene asegurado el despido.

Cuando los teleoperadores adquieren las competencias necesarias y están a pleno rendimiento, la empresa usuaria del servicio está aprovechando las capacidades intelectuales de cada una de esas personas sin necesidad de contratarlas directamente, y son muchos los cerebros que están al servicio de un objetivo. Un teleoperador bien formado puede prestar un servicio con excelencia y rapidez, porque tiene a su disposición prácticamente todos los recursos de la empresa. Para los clientes también tiene importantes ventajas porque con una sola llamada telefónica pueden acceder a todos los servicios.

Por último, hay que recalcar la relevancia del capital intelectual que aportan los teleoperadores, porque son el vínculo directo con los clientes. La red que hemos presentado es nominalista, si fuera realista tendríamos que incluir los vínculos de los teleoperadores con los clientes, es decir, el número de llamadas promedio que atienden en un día. Si se estipula una llamada cada 6 minutos, son 10 a la hora y en 
una jornada de 6 horas, descontando los tiempos de descanso, se pueden realizar alrededor de 50 llamadas. Es evidente que con este añadido los teleoperadores son los nodos centrales de la red, porque cada uno tiene al menos 50 vínculos, un número muy superior al que tienen los demás actores de la red.

\section{CONCLUSIONES.}

En este artículo se ha explicado la estructura de la organización del trabajo del sector del telemarketing con el método denominado análisis de redes sociales. A través de uno de sus conceptos, la equivalencia estructural, se ha podido descubrir una de las vulnerabilidades de la organización de este sector, la duplicidad de funciones de tres tipos de actores y los problemas de poder que pueden existir entre ellos. Una segunda vulnerabilidad encontrada es el deficiente modo de formar a los teleoperadores, lo cual tiene importantes disfunciones en la comunicación interna, así como la disminución de la calidad del servicio, especialmente durante las primeras semanas tras la incorporación de los teleoperadores a la plataforma.

Se pueden obtener dos conclusiones de este estudio. La primera es la importancia estratégica que tiene para las empresas descubrir a sus actores clave y su interacción con los demás para determinar si hay deficiencias en la línea de comunicación u organizativas. En este caso el método del análisis de redes sociales muestra su utilidad, aunque es necesario aportar la información cualitativa para completar el análisis. Los números por sí solos no explican todo.

La segunda conclusión es que para mejorar las condiciones de trabajo y la calidad del servicio habría que comenzar modificando el sistema formativo. Formación y productividad van íntimamente unidos. Los teleoperadores deberían tener más horas de práctica antes de comenzar a recibir llamadas y poder estudiar con los documentos de las empresas. De esa manera se podría evitar -o al menos reducir- las deficiencias en la comunicación interna y los errores de proporcionar información incorrecta a los clientes.

Por último, una reflexión final. A pesar del avance de la tecnología y la reducción de sectores tan ampliamente usados en el pasado como es la banca (se está sustituyendo la relación personal de los clientes con el banco, por la relación de los clientes con las aplicaciones informáticas), no parece que el factor humano vaya a desaparecer en el ámbito empresarial y laboral. El telemarketing, entonces, puede ser un intermediario válido en la relación entre el cliente, la empresa y las máquinas. Habría que incidir en la formación y la mejora de las condiciones laborales.

\section{REFERENCIAS.}

Alfaro Escolar, M. \& Alfaro Beltrán, F. (1991). El mando intermedio: figura clave en la empresa. (No. 658.43.A385). Marcombo. Barcelona. 
Paniagua López, J.

Actores clave y formación en el sector del telemarketing. Un estudio de caso aplicando el análisis de redes sociales

Álvarez, A. (2012). La introducción del teletrabajo en la administración general del Estado. Mejoras profesionales y personales. Sociología del Trabajo, 75, 73-92.

Eraso, A. B. (2002). El control del tiempo de trabajo en el teletrabajo itinerante. Sociología del Trabajo, 45, 69-96.

Borgatti, S. P. \& Everett, M. G., \& Freeman, L. C. (2002). UCINET VI for windows. Software for Social Network Analysis. User's Guide. Harvard, MA: Analytic Technologies Inc.

Borgatti, S. (2013). El problema del actor clave. Redes. Revista hispana para el análisis de redes sociales, $24(2), 1-20$.

Braga, R. (2006). Infotaylorismo: o trabalho do teleoperador e a degradação da relação de serviço. Revista Eletrônica Internacional de Economía Política da Infromaçao, da Comunicaçao e da Cultura, 8(1).

Braverman, H. (1978). Trabajo y capital monopolista. La degradación del trabajo en el siglo XX. Nuestro Tiempo.

Calderón, J. (2006). Repensar la cuestión de la resistencia en el trabajo, o buscando al trabajador perdido. Un estudio en caso en el sector del telemarketing. Sociología del Trabajo, 56, 33-74.

Cuervo, A. (1995). La dirección estratégica de la empresa. Dirección de empresas de los noventa. Editorial Civitas, Madrid.

Del Bono, A. (2000). Call Centers, ¿el trabajo del futuro? El caso de Estrategias Telefónicas, S.A. (ESTRATEL). Sociología del Trabajo, 39,3-32.

Del Bono, A. (2006). Deslocalización extraterritorial de empleos del sector servicios. Sentidos y transformaciones del trabajo. Sociología del Trabajo, 56, 3-32.

Doeringer, P. \& Piore, M. (1985). Mercados internos de trabajo y análisis laboral. Ed. Ministerio de Trabajo y Seguridad Social. Madrid.

Grant, R. (1996). Dirección Estratégica. Conceptos, Técnicas y Aplicaciones. Civitas, Madrid.

Lara Rodríguez, A. (2003). El telemarketing en España. Materiales para una cartografía del mundo del trabajo contemporáneo. Sociología del Trabajo, 49, 27-60.

López Carrasco, C. (2017). Intensificación del trabajo y tensiones del reconocimiento: experiencias del estrés de trabajadores jóvenes en los sectores del telemarketing y la consultoría. Tesis doctoral dirigida por Elena Casado Aparicio y Mario Domínguez Sánchez-Pinilla. Universidad Complutense de Madrid. 
Paniagua López, J.

Actores clave y formación en el sector del telemarketing. Un estudio de caso aplicando el análisis de redes sociales

Mintzberg, H. (2002). La estructuración de las organizaciones. Editorial Ariel, Barcelona.

Montes Cató, J. (2005). La configuración del poder en los espacios de trabajo, dispositivos disciplinarios y resistencia de los trabajadores. Sociología del Trabajo, $54,73-100$.

Ortega Olivares, M. (2014). Ética del trabajo duro y productividad en Teléfonos de México (TELMEX). Sociología del Trabajo, 80, 50-68.

Pena, P. \& Cardim, A. \& Araújo, M. (2011). Taylorismo cibernético e Lesões por Esforços Repetitivos em operadores de telemarketing em Salvador-Bahia. Cad. CRH., 24(01), 133-153.

Pérez, U. (2014). La Sección Sindical: autogestión en la acción sindical. Una mirada desde la experiencia de una sección del telemarketing. Libre Pensamiento, 78, 42-49.

Porter, M. (1982). Estrategia Competitiva. C.E.C.S.A., México.

Rimbau, E. (2002). Estrategia, costes, operaciones y recursos humanos, las variables de la oportunidad en el trabajo temporal. Capital humano: revista para la integración y desarrollo de los recursos humanos, 158, 6-15.

Saez, E. (2004). Transformaciones en la figura del profesional a través del teletrabajo. Sociología del Trabajo, 51, 61-82.

Steward, T. (1997). La nueva riqueza de las organizaciones: el capital intelectual. Granica.

Wasserman, S. \& Faust, K. (1997). Social Network Analysis. Methods and Aplications. Cambridge Universiti Press.

\section{AUTOR}

\section{Julián Antonio Paniagua López.}

Profesor de la Universidad de Valladolid. Es especialista en sociología del trabajo y análisis de redes sociales. Es autor de la monografía titulada Las dos racionalidades. Estudio antropológico de una empresa textil, que fue premiada con el primer premio de investigación del Consejo Económico y Social de la Comunidad de Madrid. Asimismo, publicó un manual para estudiantes en el cual explicaba la teoría de grafos y el software UCINET para el estudio de redes sociales. 